\title{
Vínculos y diferencias entre la escritura académica y la escritura profesional en una carrera técnica universitaria
}

\section{Articulation and differences between academic writing and professional writing in a university technical career}

Recibido: 13 de mayo de 2019 | Aprobado: 11 de junio de 2019

\section{Resumen}

En este artículo exponemos los resultados de un estudio cuyo objetivo se orientó a describir la relación -vínculos y diferencias- entre las prácticas de escritura académicas y las prácticas de escritura profesional en la carrera Técnico Superior Universitario en Electromedicina. Teóricamente nos basamos en el concepto de género discursivo desde una perspectiva social (Bazerman, 2012; Miller, 1994; Russell, 1997); nos amparamos en la investigación cualitativa (Goetz y LeCompté, 1988; Bernal, 2010) de tipo exploratoria y descriptiva (Taylor y Bogdan, 1992; García, 2012), los participantes fueron docentes, estudiantes, una estudiante que cursaba sus pasantías y un profesional técnico. Como instrumentos de recolección de datos, nos servimos de la entrevista y del cuestionario. Aunque los hallazgos indican que los géneros producidos en el pregrado difieren del género que circula en el contexto laboral, no existe una ruptura o un vacío entre ambos contextos, pues las prácticas y exigencias en la escritura académica crean un vínculo provechoso para aprender y emprender las nuevas prácticas y los nuevos géneros en el lugar de trabajo.

Palabras clave: escritura académica; escritura profesional; géneros académicos; géneros profesionales; carrera técnica universitaria.

\section{Abstract}

In this article, we present the results of a study whose objective was to describe the relationship -links and differences- between academic writing practices and professional writing practices in the Advanced University

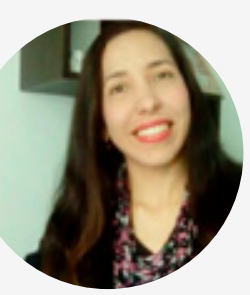

ROSMAR GUERRER0-TREJ0*

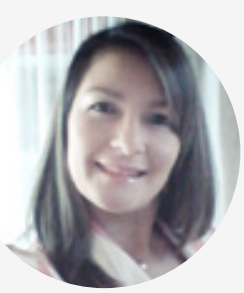

GUSMARY MÉNDEZ-CHACÓN**

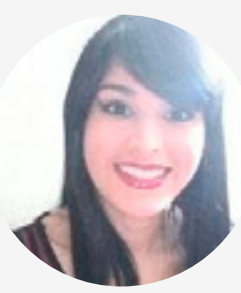

GLENDY SUÁREZ***

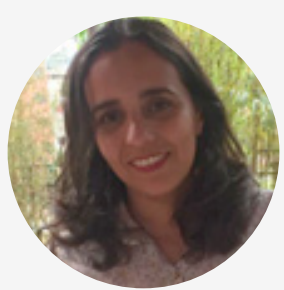

MILAGROS FARFÁN DE ROJAS****

\footnotetext{
* M Sc. Educación, mención Lectura y Escritura; Licenciada en Letras; Profesora en el área de enseñanza de la lectura y la escritura; Departamento de Botánica y Ciencias Básicas; Facultad de Ciencias Forestales y Ambientales; Universidad de Los Andes - Venezuela. Para contactar a la autora: rosmarguerrero@gmail.com

** Especialista en la Promoción de la Lectura y Escritura; M Sc. Formación del Profesorado, Licenciada en Educación; Profesora del área de Formación; Docente y Práctica Profesional; Departamento de Pedagogía; Núcleo Universitario Dr. Pedro Rincón Gutiérrez; Universidad de Los Andes - Venezuela. Para contactar a la autora: gusmarycm@gmail.com

*** Ingeniero Electrónico; Profesora en el área de Electromedicina; Departamento Ingeniería Electrónica; Núcleo de Instrumentación y Control; Universidad Nacional Experimental del Táchira - Venezuela. Para contactar a la autora: glendagmara@gmail.com

**** M Sc. Educación, mención Lectura y Escritura; Licenciada en Letras, Licenciada en Educación; Profesora en el área de enseñanza de la lectura y la escritura; Departamento de Botánica y Ciencias Básicas; Facultad de Ciencias Forestales y Ambientales; Universidad de Los Andes; Venezuela. Para contactar a la autora: derojasmila@gmail.com
}

ISSN (en línea): 1814-4152 / Sitio web: http://cuaderno.pucmm.edu.do CóMO CITAR: Guerrero, R., Méndez, G., Suárez, G. y Farfán, M. (2019). Vínculos y diferencias entre la escritura académica y la escritura profesional en una carrera técnica universitaria. Cuaderno de Pedagogía Universitaria, Vol. 16, n. ${ }^{\circ}$ 32, julio-diciembre, pp. 35-47 
Technician course in Electromedicine. Theoretically, we rely on the concept of discursive gender from a social perspective (Bazerman, 2012, Miller, 1994, Russell 1997), and we based on qualitative research and (Goetz \& LeCompté, 1988; Bernal, 2010) of exploratory and descriptive type (Taylor 1992, Garcia, 2012), the participants were teachers, students, and a female student who was in and internship, and a technical professional. As instruments of data collection, we use the interview and the questionnaire. Although the findings indicate that the genres produced in the undergraduate program differ from the genres that circulate in the labor context, it does not exist a rupture, or a gap, between both contexts, since the practices and demands of academic writing, creates a useful link to learn and undertake the new practices and new genres in the workplace.

Key words: academic writing; professional writing; academic genres; professional genres; technical career.

\section{Introducción}

En la universidad los textos escritos son una herramienta esencial para la formación académica y profesional de los estudiantes; de esta manera, resulta importante orientar la enseñanza de estos en una didáctica basada en los géneros discursivos (Navarro, 2018). Esto implica, por una parte, el conocimiento de los diversos géneros que circulan en una disciplina y su enseñanza explícita desde cada asignatura; y por otra, reconocer la función epistémica de la escritura (McLeon y Miraglia, 2001; Wells, 1990; Olson, 1998; Miras, 2000; Scardamalia y Bereiter, 1992; Serrano, 2014; Craig, 2013) y su función comunicativa y social (Bazerman, Little, Bethel, Chavkin, Fouquette, Garufis, 2005; Russell, 2002; Russell, 2013). En virtud de esto, se destaca la actuación del docente especialista como conocedor experto de la disciplina, y, por ende, de los diversos géneros discursivos que se producen en ella, de sus propósitos y objetivos que lo llevan a solicitárselos a los estudiantes.

La enseñanza de la escritura debe hacerse de manera contextualizada y situada, es decir, en cada asignatura, y por tanto concierne a todos los docentes. Alfabetizar académicamente apunta a dos objetivos: enseñar a participar en los géneros propios de cada disciplina y enseñar prácticas de escritura para aprender de ellas (Carlino, 2013). Esto demuestra la trascendencia de escribir en cada disciplina, pues no solo los estudiantes aprenderán realmente los contenidos de la asignatura, sino que también se perfilarán como escritores competentes de los textos disciplinares.

\section{Los géneros discursivos académicos y profesionales}

Cada sociedad organiza el uso de la lengua mediante los géneros discursivos (Bazerman, 2012). Estos son "eventos comunicativos" con propósitos comunicativos insertos en una comunidad discursiva (Swales, 1990); asimismo, son acciones sociales (Miller, 1994) y un sistema de actividad situada (Russel, 1997); son una herramienta social con fines sociales (Bazerman, 2012); son reconocibles por la comunidad, tienen objetivos y temáticas (estructura, gramática y vocabulario específico) dirigidas a los participantes de dichas comunidades; son predecibles y varían según el escritor o hablante, entorno y momento histórico y social (Navarro, 2018, p. 13). En síntesis, Bazerman (2012) define los géneros discursivos como:

Fenómenos de reconocimiento psicosocial que son parte de los procesos de las actividades socialmente organizadas (...). Los géneros surgen dentro de procesos sociales de gente que trata de entenderse entre sí lo suficiente para coordinar actividades y compartir significados con propósitos prácticos. (p. 133)

En los distintos espacios académicos y profesionales se crean discursos y géneros escritos propios, que parten del contexto, convenciones y situaciones reconocidas por sus miembros. En la formación universitaria los estudiantes escriben diversos géneros determinados por los propósitos y necesidades de cada disciplina y asignatura en particular; y este modo en que se produce la escritura se conoce como escritura académica. Por su parte, la escritura profesional se reconoce como aquellos géneros discursivos que se producen en instituciones públicas y privadas, determinados igualmente por las convenciones y propósitos de la comunidad en la que circulan. Se diferencian, según el ámbito, entre géneros profesionales no académicos y géneros profesionales académicos. (Navarro, 2012).

Dado que el objetivo de la educación universitaria es encauzar a los estudiantes al mundo del trabajo profesional, la escritura es una de las prácticas que se realiza para este fin, bien sea para prepararlos a escribir géneros académicos y científicos durante el pregrado y su continuidad como profesional en 
ejercicio, si laboran en una institución académica; o para hacer investigación no científica (pues no se publica) en industrias o empresas (Russell y Cortes, 2012, p. 4-5), así como la producción de diversos géneros escritos cuya esencia depende del discurso disciplinar o técnico aprendido, en principio, en la formación académica.

Los géneros académicos están circunscritos a contextos determinados, con estrategias retóricas específicas. Son componentes textuales fundamentales en la formación de los estudiantes universitarios. La construcción de saberes especializados (que gradualmente irán en ascenso) así como la apropiación de nociones y estrategias específicas para aprender en la universidad (Carlino, 2003) posibilita participar en la cultura discursiva de determinadas disciplinas y esto solo se logra a través de prácticas auténticas de los géneros académicos. (Navarro, 2012).

Por su parte, los géneros profesionales son el "conjunto de géneros discursivos que llevan a cabo los objetivos específicos de las organizaciones donde circulan" (Navarro, 2012, p. 1297), son aquellos que constituyen prácticas discursivas ya estandarizadas y reguladas institucionalmente. La función del género profesional es entonces comunicar cuestiones que involucran más el hacer (cf. Parodi, 2008). No en vano Bazerman (1988) afirmó que "un género es un constructo social que regulariza la comunicación, la interacción y las relaciones" (p. 62).

Si bien se ha reflejado en estudios realizados por Casanny y López (2010), Parodi, (2008), Parodi, Ibáñez, Venegas y González (2010), Natale y Stagnaro (2014) que entre las prácticas de escritura en la universidad y la escritura profesional existe un divorcio, justificando que la primera ejecuta insuficientes experiencias de escritura de la vida laboral, otros estudios han demostrado iniciativas que dan cuenta que la escritura en las disciplinas se han concentrado en desarrollar prácticas conducentes a la escritura de cada campo de estudio (Natale y Stagnaro, 2014), es decir, la creación de un vínculo como eje articulador entre ambas escrituras.

La creación de este vínculo obliga a superar la brecha existente entre estos ámbitos a través de las siguientes acciones: comprender lo que ocurre en lo interno de cada campo profesional (Natale y Stagnaro, 2014), identificar cuáles son los géneros académicos similares a los profesionales para aprender y analizar sus características discursivas de cada contexto (organización textual, rasgos estilísticos, consignas, formas de relacionar el contenido, propósitos de escritura, autor-lector, función, entre otros). Integrar estas acciones permite la identidad con el manejo de los textos (Casanny y López, 2010) y la posible creación de otros tipos de textos que resultan nuevos dadas las dinámicas de cada contexto. La comprensión de todos estos elementos fortalece el vínculo entre la escritura académica y la escritura profesional.

\section{Contexto de la Investigación}

En el contexto venezolano, el sistema educativo comprende dos subsistemas: el subsistema de educación básica y el subsistema de educación universitaria. Este último está estructurado en dos niveles: pregrado y postgrado. En el pregrado la formación profesional permite dos opciones, una de estas es la denominada Técnico Superior Universitario (en adelante TSU), también conocida como carrera técnica; es considerada un grado superior al bachillerato y menor a la licenciatura o ingeniería, entre otros. El TSU es un profesional capacitado con reconocida formación científica y tecnológica tanto en los aspectos teóricos como prácticos. La duración del estudio universitario técnico no excede de seis semestres y, en función del programa curricular, culmina con una pasantía profesional y trabajo de grado.

Esta investigación se ubica en el escenario académico y profesional de la carrera TSU en Electromedicina, ofrecida a la población por la Universidad Nacional Experimental del Táchira-Venezuela (en adelante UNET). Es una formación técnica a nivel universitario vinculada a las ciencias de la salud, destinada al cuido del ser humano, a través del estudio teórico y técnico de equipos médicos a partir de los principios de la tecnología sanitaria (planificación, mantenimiento, operatividad y control de calidad). La carrera TSU en Electromedicina se caracteriza por ser de naturaleza práctica según la fundamentación curricular y justificación de los profesionales encargados de su desarrollo y promoción. La concepción inicial de la naturaleza práctica de las carreras técnicas conduce a la desestimación de la enseñanza y aprendizaje explícitos de la escritura como herramienta esencial para construir conocimiento y para comunicarse en una comunidad discursiva específica (Duque, 2010; Duque y Serrano, 2012; Guerrero, Suárez y Bravo, 2017). 
En función de lo expresado anteriormente, la investigación llevada a cabo por Guerrero, Suárez y Bravo (2017) nos confirma que, aunque "los profesores especialistas desconocen que la lectura y la escritura representan la vía más idónea de comunicación, construcción y transformación del conocimiento" ( $p$. 213), entre sus resultados también se revela que "la lectura y la escritura son protagonistas e indispensables en la enseñanza y aprendizaje disciplinar en la carrera TSU en Electromedicina" (p. 213), por lo tanto, surge la iniciativa de investigar y profundizar mediante cuáles géneros se lleva a cabo la escritura en la formación académica y profesional de esta carrera. Es importante mencionar que en Venezuela son escasas las investigaciones sobre escritura en el nivel técnico universitario, por esta razón consideramos que este artículo aporta hallazgos importantes que ofrecen elementos teóricos y prácticos para continuar los estudios, y en particular, para resignificar los procesos de enseñanza y aprendizaje en este nivel académico.

El objetivo general de este artículo se orienta a describir la relación -vínculos y diferencias- entre las prácticas de escritura académicas y las prácticas de escritura profesional en la carrera TSU en Electromedicina. Los objetivos específicos son identificar cuáles géneros escriben los estudiantes de la carrera TSU en Electromedicina; identificar cuáles géneros circulan en el ámbito profesional de la TSU en Electromedicina; determinar cuáles son los propósitos de escritura de los géneros académicos y determinar cuáles son los propósitos de escritura de los géneros profesionales.

\section{Metodología}

Con miras a comprender e interpretar una realidad, la presente investigación es cualitativa (Goetz y LeCompté, 1988; Bernal, 2010) de tipo exploratoria y descriptiva (Taylor y Bogdan, 1992; García, 2012). El escenario de la investigación contó con la participación de 40 informantes (muestreo no probabilístico, ver tabla 1).

La recolección de los datos se llevó a cabo bajo la aplicación de dos instrumentos: una entrevista en profundidad y un cuestionario. La entrevista en profundidad estuvo conformada por tres guiones aplicados a los docentes, estudiante practicante y TSU en ejercicio profesional (Ver anexos). El cuestionario, diseñado con preguntas abiertas (17 ítems), fue aplicado a los 31 estudiantes. Los instrumentos de recolección de datos fueron validados previos a su aplicación por dos profesionales de la enseñanza de la lengua y metodología de la investigación (juicio de expertos). La recogida de los datos comprendió el lapso marzo/2017-abril/2018. Las entrevistas se administraron de manera oral, con soporte en audio (transcritas posteriormente para su respectivo análisis). Para darle mayor rigor y claridad a los datos del cuestionario urgió la comunicación con docentes especialistas y algunos estudiantes para precisar información difusa (encuentros informales y correo electrónico). Cabe señalar que estos instrumentos fueron diseñados tomando en cuenta objetivos y propósitos más generales y amplios de los que presentamos en este artículo; la riqueza de los datos obtenidos ha permitido usar las entrevistas y el cuestionario para otros productos de investigación (Ver Guerrero, Suárez y Bravo, 2017; Guerrero-Trejo, Suárez y Lara, en prensa).

De la entrevista a los docentes nos interesó conocer qué textos solicitan escribir a sus estudiantes y cuál es el propósito de escritura; y a la estudiante pasante y al TSU en Electromedicina, cuáles textos deben escribir como parte de sus actividades

Tabla 7. Informantes

\begin{tabular}{|l|l|}
\hline \multicolumn{1}{|c|}{ INFORMANTES } & \multicolumn{1}{c|}{ CARACTERÍSTICAS } \\
\hline $\begin{array}{l}\text { 31 estudiantes } \\
\text { Carrera TSU en Electromedicina - UNET }\end{array}$ & $\begin{array}{l}\text { Tercer semestre (10 estudiantes); cuarto semestre (8 estudiantes) y quinto semestre } \\
\text { (13 estudiantes). }\end{array}$ \\
\hline $\begin{array}{l}\text { Una estudiante en proceso de pasantía } \\
\text { (Práctica Profesional) }\end{array}$ & $\begin{array}{l}\text { Pasantías profesionales; dos meses de experiencia en un centro de salud privado } \\
\text { (San Cristóbal/Venezuela) }\end{array}$ \\
\hline Siete docentes & $\begin{array}{l}\text { Especialistas en la disciplina de la carrera TSU en Electromedicina. En su mayoría son } \\
\text { Ingenieros Electrónicos con maestría y doctorado en esta área. Para este artículo, los } \\
\text { identificamos con el código formado por "D" y un número (1 al 7) según el orden de } \\
\text { aplicación del instrumento. }\end{array}$ \\
\hline Un TSU en Electromedicina & $\begin{array}{l}\text { Ejerce la profesión; para el momento cuenta con cinco años de experiencia profesio- } \\
\text { nal en un centro de salud privado (San Cristóbal/ Venezuela) }\end{array}$ \\
\hline
\end{tabular}


laborales en la clínica y cuál es el propósito de esa producción textual. Por su parte, se destaca que, del cuestionario aplicado a los estudiantes se analizaron los ítems 1 y 4 centrados en conocer cuáles textos les solicitan los docentes en el desarrollo de sus clases y cuál es la estructura de estos.

\section{Análisis e interpretación de los resultados}

El análisis de los resultados se hizo siguiendo las siguientes proposiciones que emergen de los objetivos de este estudio: 1) Géneros académicos que escriben los estudiantes en la carrera de TSU en Electromedicina; 2) Géneros profesionales que se escriben en lugar de trabajo del TSU en Electromedicina; 3) Propósitos de la escritura en el pregrado y, 4) Propósitos de la escritura en el contexto profesional.

\section{Géneros que escriben los estudiantes en la carrera de TSU en Electromedicina}

En este apartado se da cuenta de los hallazgos encontrados en el cuestionario aplicado a los estudiantes (ítems 1 y 4) y en la entrevista hecha a los docentes especialistas. De acuerdo con los datos obtenidos del cuestionario, los estudiantes señalan 10 textos que se escriben en el pregrado, ver tabla 2.

Por su parte, según las respuestas de los docentes se advierten nueve textos que solicitan a sus estudiantes, ver tabla 3.

Al cotejar los resultados, podemos ver que hay coincidencia en el género que ambos grupos mencionan con más reiteración: el informe de laboratorio. Tomando en cuenta las respuestas de los docentes, de los estudiantes y las indagaciones que hicimos a través de correos electrónicos

Tabla 2. Géneros académicos que se escriben según los estudiantes

\begin{tabular}{|l|c|}
\hline \multicolumn{1}{|c|}{ GÉNERO } & MENCIONAD0 \\
\hline Informe técnico 0 de laboratorio & 23 veces \\
\hline Ensayo & 10 veces \\
\hline Resumen & 9 veces \\
\hline Trabajo de investigación & 3 veces \\
\hline Prelaboratorio & 2 veces \\
\hline Poslaboratorio & 1 \\
\hline Prácticas de laboratorio & 1 \\
\hline Guías & 1 \\
\hline Textos descriptivos & 1 \\
\hline Proyectos & 1 \\
\hline
\end{tabular}

y conversaciones informales, advertimos que el Informe de laboratorio es un texto complejo pues su producción conlleva la escritura y revisión de otros textos como el prelaboratorio, la práctica de laboratorio y el poslaboratorio, nombrados en menor frecuencia por algunos docentes y estudiantes.

Por ejemplo, D2 señala lo siguiente:

Sí, el informe es la comparación de lo que hicieron en el prelaboratorio con el laboratorio que considero, sí hubo una falla y por qué no se pudo hacer, entonces es una comparación y un análisis experimental en texto; es lo que hacen.

Por su parte, D4 señala que el proceso de escritura del informe es el siguiente: "ellos tienen todos los fundamentos teóricos, después desarrollan la práctica y al final hacen una conclusión comparativa dependiendo del caso de qué práctica sea". Un estudiante de quinto semestre señaló que el texto que más escribe es el informe de laboratorio, y en su respuesta sobre qué partes tiene respondió: "Prelaboratorio, cálculos teóricos, desarrollo de las simulaciones, simulaciones, poslaboratorio, análisis de los resultados".

El informe de laboratorio tiene como objetivo poner a prueba la capacidad crítica y analítica del estudiante con respecto a la discusión de resultados cuantitativos y cualitativos de cada práctica. Estos resultados derivan de la toma de datos experimentales a través de simulaciones y montajes reales para el análisis del comportamiento, ya sea de sensores o equipos electrónicos, con la finalidad de estudiar su principio de funcionamiento y aplicaciones reales. Por ejemplo, D6 expresa:

Tabla 3. Géneros que escriben los estudiantes según los docentes

\begin{tabular}{|l|c|}
\hline \multicolumn{1}{|c|}{ GÉNER0 } & DOCENTE \\
\hline Informe de laboratorio & D1, D2, D3, D4, D5, D6 \\
\hline El examen o parcial & D1, D3, D4, D6, D7 \\
\hline El prelaboratorio & D2, D6 \\
\hline La práctica de laboratorio & D2, D3 \\
\hline Trabajo de investigación & D3, D6 \\
\hline La ficha técnica & D1 \\
\hline La guía didáctica & D2 \\
\hline El quiz & D3 \\
\hline El estudio de caso & D5 \\
\hline
\end{tabular}


Yo les coloco a que hagan unas tablas comparativas del valor real, que es lo que miden: el valor simulado y el valor analítico. Hacen los cálculos y luego se les pone análisis a esos resultados, que los analicen por qué pueden ser las diferencias, si las hay o si no las hay y después conclusiones y recomendaciones.

Llama la atención que cinco de los siete docentes señalan que el examen parcial es uno de los textos que deben escribir sus alumnos, pero estos no lo mencionan ni una vez. En conversaciones informales con los estudiantes pudimos apreciar que no se menciona este género porque allí no hay desarrollo de la escritura (en el apartado teórico del examen parcial se limita a resolver las modalidades: completación y selección múltiple). El examen parcial es un género que circula asiduamente en esta universidad, se produce en el aula en un tiempo fijado y generalmente se divide en dos partes, una teórica (objetiva) y una práctica, al respecto, D6, señala:

Hago una parte objetiva, donde es completación, selección múltiple, esa es la parte objetiva. Las partes de desarrollo muy pocas veces lo pongo, por ejemplo "explíqueme el proceso de tal cosa”. Entonces, generalmente son problemas o cosas así de la materia.

Los estudiantes indican que escriben ensayos y resúmenes, pero los docentes entrevistados no los mencionan. Indagamos sobre esto y pudimos constatar que, en esta carrera, en efecto, se escriben estos géneros, pero en asignaturas auxiliares como inglés o metodología de la investigación. El trabajo de investigación se produce con menos frecuencia; D3 señala que es un texto que solicita a veces, y D6, que todos los semestres sus alumnos deben escribirlo.

El prelaboratorio, la práctica de laboratorio (mencionado por ambos grupos) y el poslaboratorio son géneros que se producen para incorporarse al Informe de laboratorio. La guía didáctica, como texto de escritura diseñado por el docente, es un modelo "Tipo parcial: ejercicios y razonamiento" (D6, entrevista), cuya finalidad es preparar a los estudiantes para un examen.

El quiz es un texto corto que se solicita al principio de una clase para evaluar conocimientos puntuales. La ficha técnica, según D1, es un texto corto en el que se da cuenta del resultado del mantenimiento de algún equipo médico. D5 señala que en el estudio de caso se analiza un caso de digitalización. Estos tres géneros no fueron reportados por los estudiantes; particularmente, la ficha técnica y el estudio de caso son textos que se producen en el quinto semestre, y no fueron nombrados por los estudiantes por el momento en que se aplicó el cuestionario, en la primera semana de clases. Por su parte, los docentes no mencionaron el texto descriptivo y el proyecto.

\section{Propósitos de la escritura en el pregrado}

Mediante la escritura de los géneros académicos se construyen saberes especializados, los estudiantes se apropian de nociones y estrategias específicas necesarias para insertarse y permanecer en la cultura discursiva académica (Navarro, 2012). Esta participación encierra diversas actividades prácticas como la evaluación de contenidos, el primer acercamiento a un tema mediante los apuntes o resúmenes y la apropiación del lenguaje técnico, tal como lo manifestó D1, además, cada área comporta ciertos géneros cuyos propósitos son la formación inicial del futuro profesional. Específicamente, en el pregrado se escriben diversos géneros con los propósitos que han definido los docentes expertos en el área. De esta manera, comentan que escribir ciertos géneros los prepara para el futuro trabajo profesional. D6 argumentó lo siguiente:

Ellos particularmente, si van a trabajar en un hospital ellos tienen que estar redactando informes técnicos, por lo menos si un equipo se dañó, ellos tienen que redactar qué se le dañó al equipo, cómo venía funcionando y por qué no funciona. Ellos tienen que redactarlo, porque eso es lo que va, de repente si viene una empresa externa a hacerle un mantenimiento o si van a comprar algo, eso se lo tienen que plasmar en unas ideas.

Así mismo lo expresó D2: "Sí, porque al final van a generar reportes donde estén, generar reportes, informes (...) La parte técnica también es importante".

Aun cuando el propósito de la escritura en la formación académica es acreditativo, los docentes reconocen que la escritura desarrolla al estudiante académica y profesionalmente. Estos, como conocedores de la disciplina, solicitan escribir ciertos géneros guiados con el objetivo fundamental de construir conocimientos teóricos, prácticos y procedimentales, y enfrentarse de manera competente ante un equipo médico, esto evidencia el 
valor epistémico -oculto-, la complejidad y alto nivel cognitivo que exige el texto que más se solicita: el informe técnico (cf. Guerrero, Suárez y Bravo, 2017).

\section{Géneros que se escriben en la profesión TSU en Electromedicina}

En este apartado mostraremos los hallazgos encontrados en las entrevistas realizadas a la estudiante en fase de pasantía profesional y al TSU en Electromedicina. Al indagar sobre los textos que la estudiante escribe, esta respondió "He realizado informes con los técnicos". El informe técnico, según sus declaraciones, se trata de un texto de carácter administrativo que se produce para dejar constancia de las fallas y reparaciones hechas a los equipos:

Ejemplo, la máquina de anestesia se le dañó algo o a la lámpara cialítica se le quemaron cuatro bombillos. Se solicitan cuatro bombillos y luego se entrega al arquitecto o a Gerencia para que ellos aprueben el dinero o compran los bombillos o mandan a buscar presupuesto. Cuando se cambian los bombillos se hace otro informe colocando, bueno, -ya está en funcionamiento la máquina de anestesia(Estudiante pasante, entrevista).

Para el momento de la entrevista la estudiante no había producido ningún informe por su cuenta, pero como parte de sus actividades de práctica profesional ella debía estar presente cuando los técnicos lo redactaban:

No, ellos los hacen y yo estoy ahí, ejemplo ellos me dicen, "[nombre de la estudiante], "¿cómo colocamos aquí?”, y, pues, yo les decía: "bueno debemos colocar informe, debemos colocar una fecha a mano derecha, no solo firmar".

En cuanto al TSU en Electromedicina, este señaló que durante los años que ha trabajado en su profesión ha ido consolidando lo aprendido en el pregrado con lo aprendido en su lugar de trabajo. En virtud de esto, el desempeño laboral ha ofrecido oportunidades para fortalecer conocimientos en la experiencia con los equipos médicos y más allá de esto, por ejemplo, con el desafío de emprender y aprender las nuevas y más complejas prácticas de lectura (cf. Guerrero-Trejo, Suárez y Lara, en prensa). Este profesional señaló que el género que ellos escriben es el informe, este no se circunscribe a un solo texto, y puede ser inicialmente un texto "informal o uno más formal" en función de la audiencia a la que va destinada, desde las enfermeras hasta la junta directiva de la clínica.

Con respecto a la producción escrita, el TSU señaló que el informe es un texto que él diseñó y aprendió en compañía de otro técnico con quien comparte responsabilidades, es decir, sin la orientación de un miembro más experto; este evento sucede porque: "Los géneros surgen dentro de procesos sociales de gente que trata de entenderse entre sí lo suficiente para coordinar actividades y compartir significados con propósitos prácticos" (Bazerman, 2012, p. 133) por tanto las necesidades comunicativas llevaron a estos dos profesionales a "inventarse" y establecer un género que a su llegada a la clínica no estaba tipificado (Cassany y López, 2010, 19). Sin embargo, el TSU señala que durante la carrera se apropió del discurso técnico que le ha permitido escribir y dar cuenta de una situación determinada de un equipo particular. Se infiere que este aprendizaje inicial le dio una base referente que ha permitido la apropiación de nuevas prácticas discursivas más específicas y especializadas.

\section{Propósitos de la escritura en el contexto profesional}

El informe en el contexto profesional del TSU en Electromedicina tiene como propósito principal dar cuenta del estado actual del funcionamiento de un equipo médico (mantenimiento, operatividad, corrección, entre otros); de ahí la importancia del proceso de escritura en este espacio laboral pues va acompañado de la experiencia física y contacto real con los equipos médicos. De esta manera, este género comporta diversos textos que se producen con un macropropósito: comunicar, pero varía según su audiencia.

En este sentido, se distinguen cinco tipos de informe:

\section{Informe para comunicar la falla de un equipo:} este texto, según el TSU, puede tener un carácter informal cuando va dirigido al cuerpo de enfermeras y médicos y, un carácter más formal cuando va dirigido a la Junta Directiva del centro de salud. Esta formalidad la determina el nivel de jerarquía de la audiencia y por tanto puede variar el formato físico del texto, mas no el contenido del mismo. 
2. Informe de corte administrativo: este texto, acompañado de un presupuesto, tiene como finalidad solicitar repuestos e insumos para la reparación de un equipo médico. Va dirigido a la Junta Directiva exclusivamente.

\section{Informe para dar cuenta de la reparación rea-} lizada: va dirigido a dos destinatarios de diferentes niveles como el informe para comunicar fallas.

4. Informe entre pares: este texto se produce cuando se presenta una situación de reparación más compleja y se requiere la orientación de un par experto. Al respecto, este informe maneja un discurso técnico especializado cuyo uso se circunscribe a profesionales de disciplinas afines. La entrega de este texto casi siempre se realiza vía correo electrónico, puede anexar imágenes y proponer posibles soluciones:

Dar las posibles fallas, pero si uno no consigue la falla es necesario comunicarse con alguien, para cuando llegue, uno le dice qué piensa que tiene el equipo o le dice la falla y el problema, pero esas personas están en otros estados, uno los llama y les dice qué posible fallas tiene para ver si lo pueden resolver desde allá o que ellos vengan (...) [son técnicos o ingenieros] también de electrónica, Ingenieros biomédicos del área, que son los que contratan para hacer cursos especializados afuera. (TSU en Electromedicina; entrevista)

5. Informe mensual: su propósito es comunicar el nivel de operatividad y funcionalidad ordinaria de los equipos, además incluye reparaciones, fallas, dadas de baja, mantenimiento en general.

\section{Discusión de resultados}

Al contrastar los resultados encontramos que los estudiantes de la carrera TSU en Electromedicina escriben un grupo de géneros (Bazerman, 2012) que apunta básicamente a la apropiación del conocimiento disciplinar, práctico y procedimental y a la evaluación sumativa de los aprendizajes de contenidos. Como lo muestran los hallazgos, el género que más se escribe en las asignaturas de la especialidad es el informe de laboratorio, texto complejo cuya producción implica la revisión y escritura de otros géneros. Dada la naturaleza práctica de la carrera, el informe de laboratorio se hace necesario y pertinente para la formación técnica pues implica poner a prueba la capacidad crítica y analítica del estudiante, ya que se discuten y argumentan resultados producto de pruebas prácticas con un equipo médico y ensayos matemáticos.

Los docentes justifican la necesidad de escribir ciertos géneros, como el informe de laboratorio, partiendo de las necesidades laborales de los futuros profesionales en ejercicio. No obstante, es preciso entender que "cuando los estudiantes van de la universidad al lugar de trabajo, no solo necesitan aprender nuevos géneros, sino también necesitan aprender nuevos caminos para aprender estos géneros" (Freedman y Adam, 1996, traducción propia). En este sentido, en el contexto laboral de la profesión TSU en Electromedicina encontramos que solo se escribe un género: el informe, texto de carácter administrativo que varía según la audiencia y el propósito comunicativo. Es así que puede ir desde un discurso más informal hasta uno más especializado y técnico.

Cabe destacar que en el pregrado de esta carrera el informe de laboratorio no representa el mismo género informe del lugar de trabajo, y esto se entiende por las marcadas diferencias entre los dos contextos retóricos (Cf. Freedman, Adam y Smart, 1994); no tienen los mismos propósitos (aprendizaje y evaluación/ información, requerimiento y solicitudes), la misma complejidad (estructura, secuencias textuales) ni destinatario final (docente/ cuerpo médico, enfermería, directivos, pares expertos); además de la posición/rol del autor, pues en el pregrado se escribe bajo condiciones y parámetros establecidos, mientras que en el lugar de trabajo, y específicamente en el caso del TSU en Electromedicina, no hubo factores externos o de poder que lo condicionaran a la escritura del informe, si tomamos en cuenta que él y su compañero crearon y tipificaron el género para esa clínica. Esto hace referencia a lo dinámico y cambiante de las prácticas profesionales (Cassany y López, 2010)

El informe no se enseña en el pregrado, sin embargo, los docentes declaran que la escritura del informe de laboratorio es importante por el valor de comunicar resultados, y apuntan que este texto les da a los estudiantes herramientas básicas para construir los informes propios del contexto laboral, "pues el trabajo asociado directamente a los equipos médicos que el técnico universitario maneja no queda solo en la experiencia con el objeto físico, sino que deviene en un informe o reporte" (Guerrero, Suárez y Bravo, 2017, p. 7). Además, se 
destaca que si bien, no se enseña por parte de los profesores, los estudiantes tienen la oportunidad de acercarse al género informe, una vez entran a la fase de pasantías; puede decirse que esta etapa del estudio es el eslabón entre lo netamente académico y lo netamente laboral. La estudiante pasante entra a la comunidad de práctica de su futura profesión (Wenger, 1998), por tanto, el conocimiento del género, ya tipificado en esa clínica, se hace a partir y a través de la práctica situada, de la cooperación y enseñanza explícita por parte de los expertos.

En virtud de esto, corroboramos que los géneros que se escriben en el pregrado de TSU en Electromedicina difieren del género que se escribe en el contexto laboral (Cf. Cassany y López; Freedman, Adam y Smart, 1994). Si bien no hay una transición entre el formato de ambos géneros o estructuras y propósitos retóricos, y ni siquiera, en el pregrado hay una escritura del informe como simulación, podemos señalar que no existe una ruptura o un vacío entre ambas prácticas de escrituras; pues la producción del informe de laboratorio es un vínculo -inicial- con la construcción del conocimiento profesional; su desarrollo y todo lo que implica llegar al producto textual conlleva que los estudiantes piensen y actúen como técnicos en ejercicio, resuelvan posibles problemas, analicen, argumenten y comuniquen sus resultados. En este espacio hay un encuentro entre la escritura académica y la escritura profesional, lo que se aprende en el pregrado mediante las prácticas de lectura y escritura, sin duda, es un nexo esencial entre ambos mundos.

\section{Conclusiones}

Este es un primer acercamiento al estudio sobre las prácticas de escritura académica y escritura profesional en la carrera TSU en Electromedicina y los géneros que se escriben en ambos contextos. Hemos advertido que, en efecto, ciertas prácticas y exigencias en la escritura y la naturaleza técnica de la carrera crean un vínculo conveniente para aprender los géneros profesionales. La inserción de los estudiantes en su futura comunidad de práctica (Wenger, 1998) dependerá de la apropiación de dicha práctica y esto solo es posible si se dispone "de los artefactos escritos necesarios para llevarla a cabo" (Cassany y López, 2010, p. 13). Es así que:

La centralidad de los géneros académicos, dentro de este continuum, como ejes articulatorios entre los profesionales y otros géneros especializados (e.g., géneros científicos) revela su carácter fundamental en la construcción de un sello disciplinar especializado. Así, este espacio académico de formación actúa como una guía conductora inicial, ofreciendo un repertorio de géneros que se constituyen en accesos al conocimiento y a las prácticas especializadas escritas, es decir, al saber y al hacer (Parodi, 2008, p. 31).

Diversas investigaciones señalan algunas divergencias entre la escritura en la universidad y en el lugar de trabajo (Cassany y López, 2010; Dias, Freedman, Medway y Pare, 2011; Freedman y Adam, 1996; Freedman, Adam y Smart, 1994; entre otros), pero asimismo reconocen ciertos puntos de encuentro entre el aprendizaje de los géneros académicos y los profesionales. Estamos de acuerdo en ello, tomando en cuenta nuestros hallazgos que niegan un vacío abrupto entre ambos contextos. No obstante, la invitación no es a tomar posturas radicales respecto a estos eventos ni al andamiaje que debe haber entre la universidad y el campo laboral, sino recabar suficiente información respecto a esta transición, que permita dar sugerencias y alternativas que en definitiva beneficien a los estudiantes.

En este estudio asumimos una posición teórica del género enfocada en el contexto social (Bazerman, 2012; Miller, 1994; Russel, 1997; Swales, 1990) (véase Guerrero-Trejo, Suárez y Lara, en prensa), sin embargo, consideramos que los géneros también deben estudiarse desde una perspectiva más lingüística y textual, lo que sin duda resultaría en un conocimiento más integral; esto queda pendiente para una futura investigación. Es importante, asimismo, estudiar cómo se desarrollan la lectura y escritura en la carrera TSU en Electromedicina, según las perspectivas de los estudiantes; pues Guerrero, Suárez y Bravo (2017) señalan que los docentes de esta carrera poseen una creencia desacertada con respecto a la enseñanza y aprendizaje de estos procesos. Creemos firmemente que analizar la lectura y escritura académicas desde diversos enfoques teóricos y metodológicos permitirá comprenderlos mejor y emprender acciones en favor de la formación de los estudiantes y de los docentes.

Para cerrar, insistimos en la formación en alfabetización académica para los docentes y en el trabajo colaborativo entre el docente de lengua y el docente especialista (cf. Carlino, 2014; Guerrero, 2015; Harran, 2011; Jacobs, 2007; López y Pedraza, 
2012; Moyano, 2004; Paretti, 2011), con el fin de crear alianzas pedagógicas y espacios de investigación que apunten a robustecer y a nutrir los estudios desde la experiencia y conocimiento de expertos de distintas áreas, como es el caso de este artículo. -

\section{Referencias}

Bazerman, Ch. (1988). Shaping written knowledge. The genre and activity of the experimental article in science. Madison, WI: University of Wisconsin Press.

Bazerman, Ch., Little, J., Bethel, L., Chavkin, T., Fouquette, D. y Garufis, J. (2005). Reference Guide to Writing Across the Curriculum. West Lafayette: Parlor Press. Disponible en: http://wac. colostate.edu/books/bazerman_wac/wac.pdf

Bazerman, Ch. (2012). Géneros textuales, tipificación y actividad. México: Benemérita Universidad Autónoma de Puebla.

Bernal, C. (2010). Metodología de la investigación. Colombia: Pearson.

Carlino, P. (2003). Alfabetización académica: un cambio necesario, algunas alternativas posibles. En Educere, Vol., 16 (20), 409-420. Disponible en: http://www. saber.ula.ve/bitstream/123456789/19736/1/ articulo7.pdf

Carlino, P. (2013). Alfabetización académica diez años después. En Revista Mexicana de Investigación Educativa REDU. 18, (57), 355-381 Disponible en: http://www.comie.org.mx/revista/v2018/rmie/ index.php/nrmie

Carlino, P. (2014). Se aprende muy diferente una materia si se lee y escribe sobre sus temas. En entrevista con Serrano, S., Mostacero, R. y Duque, Y. En Serrano de Moreno, S. y Mostacero, R. (Comps.) La escritura Académica en Venezuela. Investigación, reflexión y propuestas (pp. 27-47). Mérida- Venezuela: Vicerrectorado administrativo. Universidad de Los Andes.

Cassany, D, y López-Ferrero, C. (2010). Capítulo 12. De la Universidad al mundo laboral: Continuidad y contraste entre las prácticas letradas académicas y profesionales. En Giovanni Parodi, editor. Alfabetización académica y profesional en el siglo XXI: leer y escribir desde las disciplinas. Barcelona: Planeta Ariel. p. 347-374.

Craig, L. (2013). Integrating writing strategies in EFL/ ESL University contexts. A writing- across-thecurriculum aproach. New York: Tailor y Francis.

Dias, P., Freedman, A., Medway, P. y Pare, A. (2011). Worlds Apart: Acting and Writing in Academic and Workplace Contexts. New York: Routledge.
Duque, Y. (2010). La lectura y la escritura como prácticas académicas universitarias. (Trabajo Especial de Grado de Maestría inédito). Universidad de Los Andes. Mérida - Venezuela.

Duque, Y. y Serrano, S. (2012). Las prácticas de escritura en los programas de formación técnica de emergenciólogos. En Legenda, Vol., 16 (14), 10-30. Disponible en: http://erevistas.saber.ula. ve/index.php/legenda/article/view/3917

Freedman, A. y Adam, C. (1996). Learning to Write Professionally: "Situated Learning" and the Transition from University to Professional Discourse. Journal of Businees and Technical Communication, 10 (4), 395-427. Disponible en: https://journals.sagepub. com/doi/10.1177/1050651996010004001

Freedman, A., Adam, C. y Smart, G. (1994). Wearing Suits to Class: Simulating Genres and Simulations as Genre. Written Comunication, 11 (2), 193-226. Disponible en: https://journals.sagepub.com/ doi/10.1177/0741088394011002002

García, J. (2012). Métodos y técnicas de investigación en educación. Madrid: UNED.

Goetz, J. y LeCompté, M. (1988). Etnografía y diseño cualitativo en investigación educativa. Madrid: Morata.

Guerrero, R. (2015). La escritura académica, la formación docente y el trabajo colaborativo interdisciplinario. Algunas consideraciones para su desarrollo. En Legenda, 19 (20), 57-73. Disponible en: http:// erevistas.saber.ula.ve/index.php/legenda/article/ view/6858

Guerrero, R., Suárez, G., Bravo A. (2017). Leer y escribir en una carrera técnica universitaria. En Enunciación, 22 (2), 202-216. Disponible en: http://doi.org/10.14483/22486798.12336

Guerrero-Trejo, R., Suárez, G. y Lara, J. (en prensa). Formación académica y lectura de géneros profesionales en una carrera técnica universitaria.

Harran, M. (2011). Engineering and Language Discourse Collaboration: Practice Realities. Across the Disciplines 8 (3), Disponible en: http:// wac.colostate.edu/atd/clil/harran.cfm

Jacobs, C. (2007). Towards a critical understanding of the teaching of discipline-specific academic literacies: making the tacit explicit. Journal of Education, (41), pp. 59-81. Disponible en: http://joe.ukzn.ac.za/ Libraries/No_41_2007/Towards_a_critical__of_the_ teaching_of_discipline-specific_academicliteracies_ making_the_tacit_explicit.sflb.ashx]

López, K. y Pedraza, C. (2012). Cambios en las representaciones y en las prácticas de docentes universitarios sobre la escritura a partir de una estrategia colaborativa de formación en alfabetización académica. (Trabajo de grado inédito) Universidad del Valle, Cali- Colombia. 
McLeod, S. y Miraglia, E. (2001). Writing Across the Curriculum in a Time of Change: In WAC for the New Millennium: Strategies for Continuing WritingAcross-the-Curriculum Programs. Urbana, IL: NCTE.

Miller, C. (1994). Genre as social action. En A. Freedman y A. Medway (Eds.), Genre and the New Rhetoric (pp. 23-42). London: Taylor y Francis.

Miras, M. (2000). La escritura reflexiva: aprender a escribir y aprender acerca de lo que se escribe. En Infancia y aprendizaje, (89), 65-80. Disponible en: https:// dialnet.unirioja.es/servlet/articulo?codigo $=48632$

Moyano, E. (2004). La escritura académica: una tarea interdisciplinaria a lo largo de la currícula universitaria. En Revista Texturas, 4 (4), pp. 109-120 Disponible en: http://www.ungs.edu.ar/prodeac/wp-content/ uploads/2011/08/Moyano_2004_La-escrituraacademica.pdf

Natale, L. y Stagnaro, D. (2014). Alfabetización profesional durante la carrera universitaria: entre la universidad y la empresa. En Itinerarios educativos, 7 (7). Disponible en: www.ungs.edu.ar/prodeac

Navarro, F. (2012). ¿Quéson los géneros profesionales? Apuntes teórico-metodológicos para el estudio del discurso profesional. Actas del IV Congreso Internacional de Letras "Transformaciones Culturales. Debates de la teoría, la crítica y la lingüística en el Bicentenario" (Buenos Aires, 22-27 noviembre de 2010), Publisher: Universidad de Buenos Aires, Editors: A. Cristófalo, J. Ledesma, pp.1294-1303

Navarro, F. (2018). Didáctica basada en géneros discursivos para la lectura, escritura y oralidad académicas. En F. Navarro y G. Aparicio (Eds.), Manual de lectura, escritura y oralidad académicas para ingresantes a la universidad (pp. 13-23). Bernal: Universidad Nacional de Quilmes.

Olson, R. (1998). El mundo sobre el papel: El impacto de la escritura y la lectura en la estructura del conocimiento. Barcelona: Gedisa.

Paretti, M. (2011). Interdisciplinarity as a lens for theorizing language/content partnerships. Across the Disciplines, 8 (3), Disponible en: http://wac. colostate.edu/atd/clil/paretti.cfm

Parodi, G. (2008). Géneros académicos y géneros profesionales: Accesos discursivos para saber y hacer. Valparaíso: Ediciones Universitarias de Valparaíso. Pontificia Universidad Católica de Valparaíso.

Parodi, G., Ibáñez, R., Venegas, R. y González, C. (2010). Identificación de géneros académicos y géneros profesionales: principios teóricos y propuesta metodológica. En: G. Parodi (ed.), Alfabetización académica y profesional en el siglo XXI. Chile: Ariel, pp. 249-289.

Russell, D. (1997). Rethinking Genre in School and Society: And Activity Theory Analysis. Written Communication, 14, (4), 504-554. Disponible en: https://drrussel. public.iastate.edu/at\%26genre/ at\%26genre.html

Russell, D. (2002). Writing in the Academic Disciplines. A Curricular History. Carbondale, Edwardsville: Southern Illinois University Press.

Russell, D., y Cortes, V. (2012). Academic and scientific texts: The same or different communities. En Castelló, M. y Donahue, C. (Edit.), University writing. Selves and texts in academic societies. (pp.3-18). Emerald Group Publishing Limited.

Russell, D. (2013). Contradictions regarding teaching and writing (or writing to learn) in the disciplines: What we have learned in the USA. Revista de Docencia Universitaria, 11 (1), 161-181. Disponible en: http://red-u.net/redu/index.php/ REDU/article/view/584

Scardamalia, M. y Bereiter, C. (1992). Dos modelos explicativos de los procesos de composición escrita. En Infancia y Aprendizaje, (58), 43-64. Disponible en: https://dialnet.unirioja.es/servlet/ articulo?codigo $=48395$

Serrano, S. (2014). La lectura, la escritura y el pensamiento. Función epistémica e implicaciones pedagógicas. En Lenguaje, 42 (1) 97-122. Disponible en: http://www.scielo.org.co/pdf/leng/ v42n1/v42n1a05.pdf

Swales, J. (1990). Genre analysis: English in academy and research settings. Glasgow: Cambridge University Press.

Taylor, S. y Bogdan, R. (1992). Introducción a los métodos cualitativos de investigación. España: Paidós.

Wells, G. (1990). Talk about Text: Where Literacy is Learned and Taught. Curriculum Inquiry, 20 (4), 369-405. Disponible en: https://doi.org/10.1080/ 03626784.199011076083

Wenger, E. (1998). Communities of Practice: Learning, Meaning, and Identity. Cambridge: Cambridge University Press. 


\section{Anexo 1}

\section{Guion de entrevista en profundidad aplicada a los docentes especialistas}

1. ¿Para qué sirve leer y escribir en la universidad y específicamente en su asignatura?

2. ¿Cómo se utiliza la escritura y la lectura en su asignatura?

3. ¿Durante el desarrollo de su asignatura, qué textos solicita a sus estudiantes que lean ¿Libros, guías, capítulos de libros, artículos? ¿qué tipo de artículos? ¿por qué esos y no otros?

4. ¿Por qué considera que estos textos son los indicados para enseñar su asignatura y para que sus estudiantes aprendan?

5. ¿Durante el desarrollo de su asignatura, qué textos solicita a sus estudiantes que escriban? ¿Cómo llaman a estos textos? ¿Resúmenes, exámenes, informes, ensayos, otros? ¿Cuál es su estructura? ¿Por qué esos y no otros?

6. ¿Con qué intención y finalidad solicita que sus estudiantes lean y escriban estos textos en particular? (evaluar contenido, enseñar/enseñar contenido)

7. ¿Qué piensa de las tareas de escritura que le entregan sus alumnos? Es decir, ¿qué aspectos de escritura considera/ valora y exige? ¿Cómo evalúa la escritura (redacción, ortografía, coherencia, cohesión, pertinencia, contenido)?

8. ¿Cómo evalúa la lectura? Es decir, ¿leen de forma individual en el aula o antes de la clase? ¿Usted lee el texto con ellos, lo discuten en aula, quién comienza la discusión y cómo? (lluvia de ideas, preguntas sobre la lectura) ¿Usted asigna o sugiere la lectura?

9. ¿Cómo son las consignas e instrucciones de escritura de los textos? Es decir, ¿les indica a sus estudiantes cómo lo van a redactar? ¿Cuáles son las pautas que les da? ¿De qué manera ofrece esas pautas? (por escrito o de manera oral)

10. ¿Proporciona algún tipo de ayuda para la escritura de los textos? ¿En qué momento se da esa ayuda (antes, durante o después de que los estudiantes entregan el texto o tarea)?

\section{Anexo 2}

\section{Guion de cuestionario aplicado a los estudiantes}

1. ¿Qué clase de textos (informes técnicos, ensayos, resumen, entre otros) te solicitan los profesores para escribir? Explica.

2. ¿Con qué frecuencia escribes los textos solicitados por los profesores?

3. ¿En qué lugar escribes los textos solicitados? ¿En clase? ¿Fuera de clase? Explica.

4. ¿Podrías describir las partes que contiene el texto que con mayor frecuencia te solicitan los profesores? Explica.

5. Para el caso de los textos teóricos, ¿qué fuentes de información utilizas?

6. ¿Dónde consigues las fuentes de información? biblioteca, internet, los profesores suministran la bibliografía

7. ¿Qué texto utilizas para la bibliografía? (libros, enciclopedias, revistas científicas, guía didáctica entre otros)

8. ¿Recibes orientación por parte de los profesores para acceder a las fuentes de mayor confiabilidad?

9. En el transcurso de esta carrera tecnológica, cuando un profesor solicita un texto, ¿recibes orientaciones por parte del facilitador para escribirlo? Explica.

10. ¿Cuántas veces el profesor revisa el texto solicitado antes de entregar la nota final?

11. ¿Qué dificultad presentas al momento de escribir un texto?

12. Cuando el profesor evalúa el texto final, ¿qué observaciones realiza para justificar la nota?

13. ¿Qué variedad de textos lees en clase? 
14. ¿Cuál es la finalidad de leer textos en clases, ya sea en español o inglés?

15. ¿Qué dificultad presentas al momento de leer los textos, ya sea en español o inglés?

16. ¿Recibes orientación por parte de los profesores para lectura y comprensión de los textos que utilizan?

17. ¿De qué manera el profesor evalúa la lectura que has realizado de un texto, ya sea en español o inglés?

\section{Anexo 3}

Guion de entrevista en profundidad aplicada al TSU en Electromedicina

1. ¿Hace cuánto te graduaste?

2. ¿En qué institución hiciste las pasantías?

3. ¿Cuánto tiempo tienes trabajando en esta clínica?

4. ¿Cuál es tu función en este empleo?

5. ¿Cree que lo que aprendiste en la carrera los 3 años de estudio te han servido para emplearlo aquí?

6. En este campo laboral, ¿de qué manera hacen uso de la lectura y escritura?

7. En cuanto a la lectura, ¿lo textos que lees son en español o inglés?

8. ¿La carrera te preparó para leer y escribir esos textos?

9. ¿Te sientes experto en la lectura y escritura de estos textos?

10. ¿En las pasantías hiciste lo mismo que estás haciendo en la profesión?

11. Durante el pregrado ¿tuviste la oportunidad de leer o escribir los textos que lees y escribes en la profesión?

12. ¿Qué textos recuerdas haber leído y escrito durante el pregrado que hayan ayudado para formarte?

13. Cuando empezaste en este trabajo, ¿cómo te sentiste al momento de enfrentarte con los textos que debías leer y escribir?

14. ¿Con qué motivo el TSU en Electromedicina lees los textos?

15. ¿Con qué motivo el TSU en Electromedicina escribes los textos?

16. ¿Cuál es la estructura de ese texto?

17. Cuando te solicitaron escribir ese texto, ¿quién te orientó?

\section{Anexo 4}

Guion de entrevista en profundidad aplicada a la estudiante pasante

1. ¿Cuánto tiempo tienes haciendo pasantías en esta clínica?

2. En este campo laboral ¿De qué manera hacen uso de la lectura y escritura?

3. ¿Qué textos has leído y qué textos has escrito durante las pasantías?

4. En cuanto a la lectura ¿cómo estuvo tu comprensión cuando te enfrentaste por primera vez a esos textos?

5. En cuanto a la escritura ¿cómo estaban tus habilidades para escribir por primera vez esos textos?

6. ¿Para qué el TSU en Electromedicina promueve la lectura de textos?

7. ¿Para qué el TSU en Electromedicina promueve la escritura de textos?

8. ¿Cuál es la estructura de esos textos?

9. ¿Consideras que la carrera te preparó para enfrentarse a esos textos?

10. En la parte académica, ¿qué has tenido que leer y escribir para hacer su trabajo de aplicación profesional o para rendir cuentas a la tutora o al tutor de aquí? 